\title{
A STUDY OF BLASTING RECORDED IN SOUTHERN CALIFORNIA
}

\author{
By Harry O. Wood and Charles F. Richter
}

\section{INTRODUCTION}

Seismographic registration of vibratory earth motion was begun with an experimental torsion seismometer assembly at the laboratories of the Mount Wilson Observatory in Pasadena, California, in January, 1923. Not long afterward a second assembly was put into experimental operation at the Norman Bridge Laboratory of Physics at the California Institute of Technology at Pasadena. With many small, experimental changes in design and adjustment, which led to change in constants and necessitated some interruptions, recording on an experimental basis continued for more than four years. However, in October, 1926, recording was begun with equipment of more permanent form at Riverside and, after a short time, at the Seismological Laboratory at Pasadena in March, 1927, and at Santa Barbara and at La Jolla in May, 1927. After the occupation of the Seismological Laboratory, the experimental recording at the laboratories of the Mount Wilson Observatory and at the Norman Bridge Laboratory of Physics was brought to a close in the course of a few months. In April, 1928, recording was begun at the Observatory on Mount Wilson; and in September, 1929, at Tinemaha, and at Haiwee, in the Owens Valley, Inyo County, California. The co-ordinates of these stations, and the heights above sea-level, are given in Table I. At all

TABLE I

\begin{tabular}{|c|c|c|c|}
\hline Station & $\stackrel{\lambda}{W}$ & \multicolumn{2}{|c|}{$h$-Altitude } \\
\hline Pasadena Station & & & \\
\hline Mt. Wilson Laboratory. $\left(34^{\circ} 09^{\prime} 3\right)$ & $\left(118^{\circ} 08^{\prime} .1\right)$ & & \\
\hline Seismological Laboratory $34^{\circ} 08^{\prime} \cdot 9$ & $118^{\circ} 10^{\prime} \cdot 3$ & 295 & 965 \\
\hline Mount Wilson........... $34^{\circ} \quad 13 \cdot 4$ & $118^{\circ} 03.5$ & 1,742 & 5,714 \\
\hline Riverside ........ & $117^{\circ} 22: 5$ & $250 \pm$ & 825 \\
\hline $32^{\circ} 51: 7 \pm$ & $117^{\circ} 15: 3 \pm$ & $7.7 \pm$ & 28 \\
\hline Inta Barbara & $119^{\circ} \quad 42^{\prime} 9$ & $100 \pm$ & $325=$ \\
\hline $36^{\circ} 08: 2$ & $117^{\circ} 58: 6$ & $1,110 \pm$ & 3,640 \\
\hline Tinemaha* & $118^{\circ} 15.5$ & $1,180 \pm$ & 3,870 \\
\hline
\end{tabular}

* The rock material underlying Haiwee is a loosely cemented tuff; that underlying Tinemaha is basalt, outcropping at the surface in both places. That underlying the other stations is referred to in the text of this article. 
these permanent stations improvement in the equipment has been made at intervals, especially in means for correlating the time-marks and determining the clock corrections. During the experimental operation the time-keeping was generally mediocre and at times poor, but there were some intervals of excellent time control. On the map, Figure 1, the positions of these stations are indicated by letters as follows :

A Seismological Laboratory, Pasadena, California.

The temporary station at the Mount Wilson Observatory Laboratories was about $3.6 \mathrm{~km}$. E. $14^{\circ} \mathrm{N}$. from $\mathrm{A}$.

The temporary station at the Norman Bridge Laboratory of Physics was about $4.4 \mathrm{~km}$. E. $18^{\circ} \mathrm{S}$. from A.

B Seismological Station, Riverside.

C Seismological Station, Santa Barbara.

D Seismological Station, La Jolla.

E Seismological Station, Mount Wilson.

F Seismological Station, Tinemaha.

G Seismological Station, Haiwee.

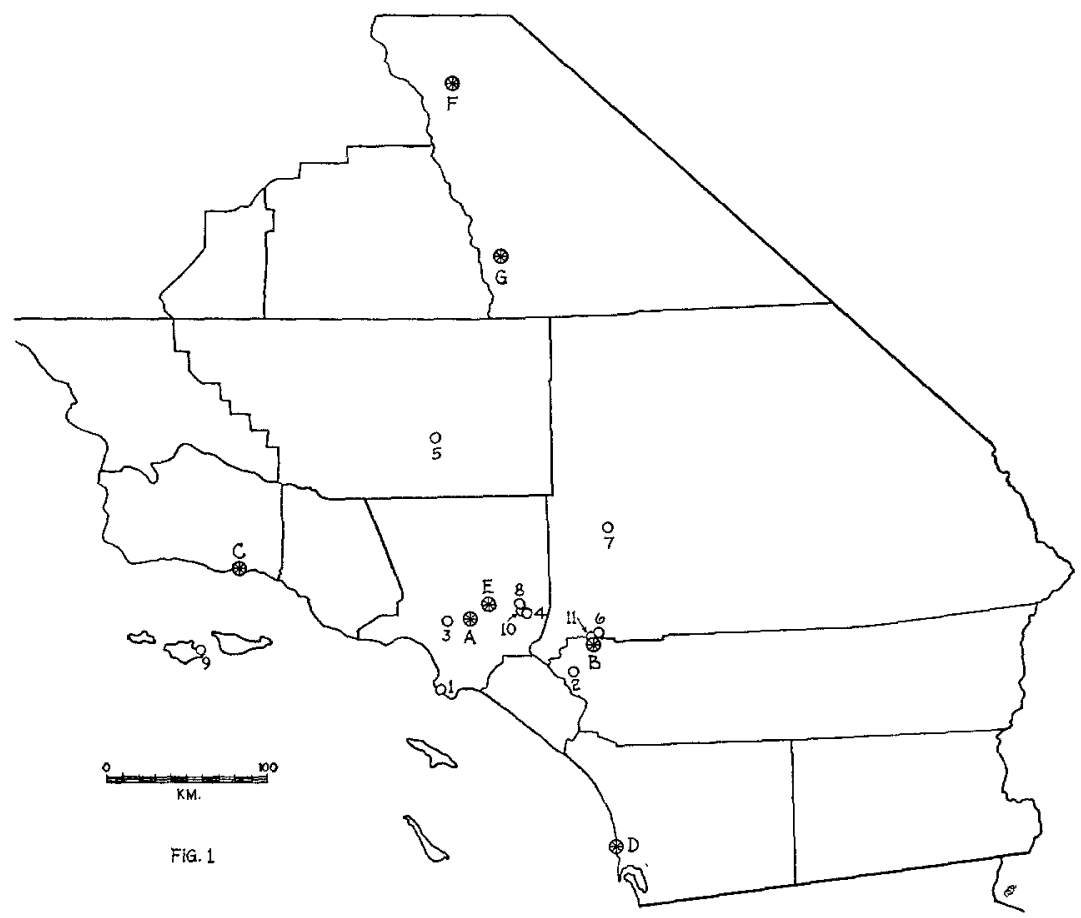

FIG. 1 


\section{Blasts}

From the beginning of registration down to October 31, 1930, blasts at eleven different places have been recorded at one or more of the stations mentioned, including three groups of three or more separate explosions. These have ranged in distance between source and station from about 3.5 kilometers (2.2 miles) to 195 kilometers (121 miles). Twenty-three different wave-paths have been involved. In five or six instances the dates of these blasts were known in advance, but in only one case, that of the great blast near Corona, has it proved possible to determine the time of firing precisely. Results of the study of these are presented below.

These blasts, and groups of blasts, are numbered and named as follows. The numbers also indicate the locations on the accompanying map, Figure 1. The order is chronological in general.

1. Palos Verdes Road

(-) (Pebbly Beach-not registered)

2. Corona Quarry

3. Hollywood Quarry

4. Big Dalton Dam

5. Monolith Quarry
6. Slover Mountain, or Colton, Quarry

7. Victorville Quarry

8. San Gabriel dam site

9. Skunk Point

10. Glendora Road

11. Crestmore Quarry

1. Palos Verdes Road.-The first blast to be recorded was set off on October 21, 1923, at a point on the coast on the southwest side of the San Pedro hills 49 kilometers ( 32 miles) from the pier of the temporary seismometer at the laboratories of the Mount Wilson Observatory. This blast was fired in connection with road building at Palos Verdes Estates; the charge was stated to consist of 100,000 pounds of black powder and 10,000 pounds of dynamite, and a very large mass of earth was dislodged and heaved into the sea. This was recorded with unexpected clearness, notwithstanding that more than 245 meters $(800+$ feet $)$ of loose, coarse wash gravel, lying at the base of the Sierra Madre range, lay directly beneath the seismometer pier, and that rocks of the younger geologic series, largely or wholly post-Miocene, and in part i11-consolidated, reach to a depth of about 3,000 meters, perhaps more, along the wave-path between source and station. The date of this blast was known in advance but useful registration was not anticipated and no effort was made to determine the time of firing.

(-) Pebbly Beach.-Of the next major blast to be set off in southern California no indication can be found on the records. The date of 
firing was known in advance and the time of detonation was watched for without result. This blast was set off on January 18, 1924, at a rock quarry near Pebbly Beach on Santa Catalina Island. The charge was stated to consist of fifty tons of explosives (dynamite). The wave-path between the station and the intersection with the coast-line was very nearly the same as in the case of the San Pedro Hills blast; between the source and the point on the coast is a sea-trough which exceeds 400 fathoms in depth at maximum.

2. Corona Quarry.-The next and largest of these blasts was set off on April 27, 1924, in a quarry operated by the Blue Diamond Company about $6 \mathrm{x} / 4$ kilometers (about 4 miles) southeast of Corona, California, distant 67.5 kilometers ( 42 miles) from the piers of the temporary seismometers at the laboratories of the Mount Wilson Observatory. The purpose of this blast was to make ready economically for the rockcrusher a huge mass of quarry rock. Three hundred and twenty-eight thousand eight hundred pounds of dynamite were packed into thirteen pockets, $12 \times 12$ feet in size, cut out of the rock along cross-cut drifts run at right angles to the inner ends of three adits cut back from the face of the quarry. These huge charges were detonated simultaneously, breaking free and shattering a mass of fresh, tough rhyolite porphyry (quartz-porphyry) estimated at 1,695,000 tons.

The date of firing of this blast was known in advance. The static magnification factors of the seismometers in experimental use at the time were more than usually large-about 3,300 in one case, 2,500 in another, and 400 in the third case-so that useful records were anticipated. Consequently arrangements were made to determine the time of firing closely. We are glad to acknowledge the courtesy of officials of the Blue Diamond Company and engineers of the Hercules Powder Company in permitting access to the firing switch at time of firing.

Four observers with watches timed the closing of the firing switch, reading seconds and estimating tenths. Because of the method employed the following discussion is included. The two better watches were compared with the clock in the Physical Laboratory of the Mount Wilson Observatory at 9:00 a.m., P.S.T., at which time this clock was compared with the radio time-signal sent from Annapolis, NSS, (at noon, Eastern Standard Time). The time-marks on the seismogram were made directly by this clock. At 9:32 a.m., P.S.T., by the clock, all four watches were compared with it. At 2:09 p.m., P.S.T., by one of the better watches, the other three were compared with it. At about 2:30 p.m., the firing switch was closed and timed by all. The shock through the earth 
was felt at once, estimated by all at less than one-tenth of a second after the closing of the firing switch. (The shock through the air, rattling frame and windows, was perceptibly later, but it was not timed.) At 5: 41 p.m., P.S.T., by the Laboratory clock all four watches were again compared with it, and at 7:00 p.m., P.S.T., the two better watches were again compared with the clock at which time it was again compared with the radio time-signal sent from Annapolis, NSS, (at 10 p.m., E.S.T.). The observations proved to be in good agreement. When reduced independently, by two different methods, by Harry O. Wood and by Dr. J. A. Anderson of the Mount Wilson Observatory, the time of closing the firing switch was determined to be $2: 30: 11.1$ p.m., P.S.T. in both cases.

It is interesting to note that the earth shock produced was registered at Pasadena (by three experimental seismometers-two at the laboratories of the Mount Wilson Observatory and one at the Norman Bridge Laboratory of Physics, all installed on loose, coarse, wash gravel more than 245 meters [800+ feet] in depth) with amplitudes noticeably greater than in the case of many natural earthquakes originating at about the same distance. However, in calling attention to this it is desirable to say also that such natural earthquakes are so small in energy that they can be detected only by sensitive physical instruments such as those designed especially to record them. In other words, the shock from this blast, though greater than many local earthquakes, was notwithstanding too small to produce ordinarily perceptible effects outside a very small area probably included for the most part within the limits of the quarry. The disturbance propagated through the air was perceptible at considerably greater distance.

In this case the shock-waves originated in fresh, tough quartz porphyry which rests upon granite at no great depth, possibly passing by gradation from one facies to the other. En route from the source to the seismometers the waves passed through, or beneath, a folded body of Tertiary rocks. The vertical thickness of this body is unknown. It is almost certain, however, that the waves entered the unconsolidated gravels, more than 245 meters $(800+$ feet) in vertical thickness, beneath the temporary stations at Pasadena, from an underlying body of granitic rock. Thus there is the possibility that granitic material forms all, or a principal part, of the path traversed.

The locations of these blasts, and of the others described below, are shown on the outline map, Figure 1. Distances, time-intervals, etc., are exhibited in tabulations included below, Tables II and III. 
A TUDY OF BLASTING RECORDED IN SOUTHERN CALIFORNIA 33

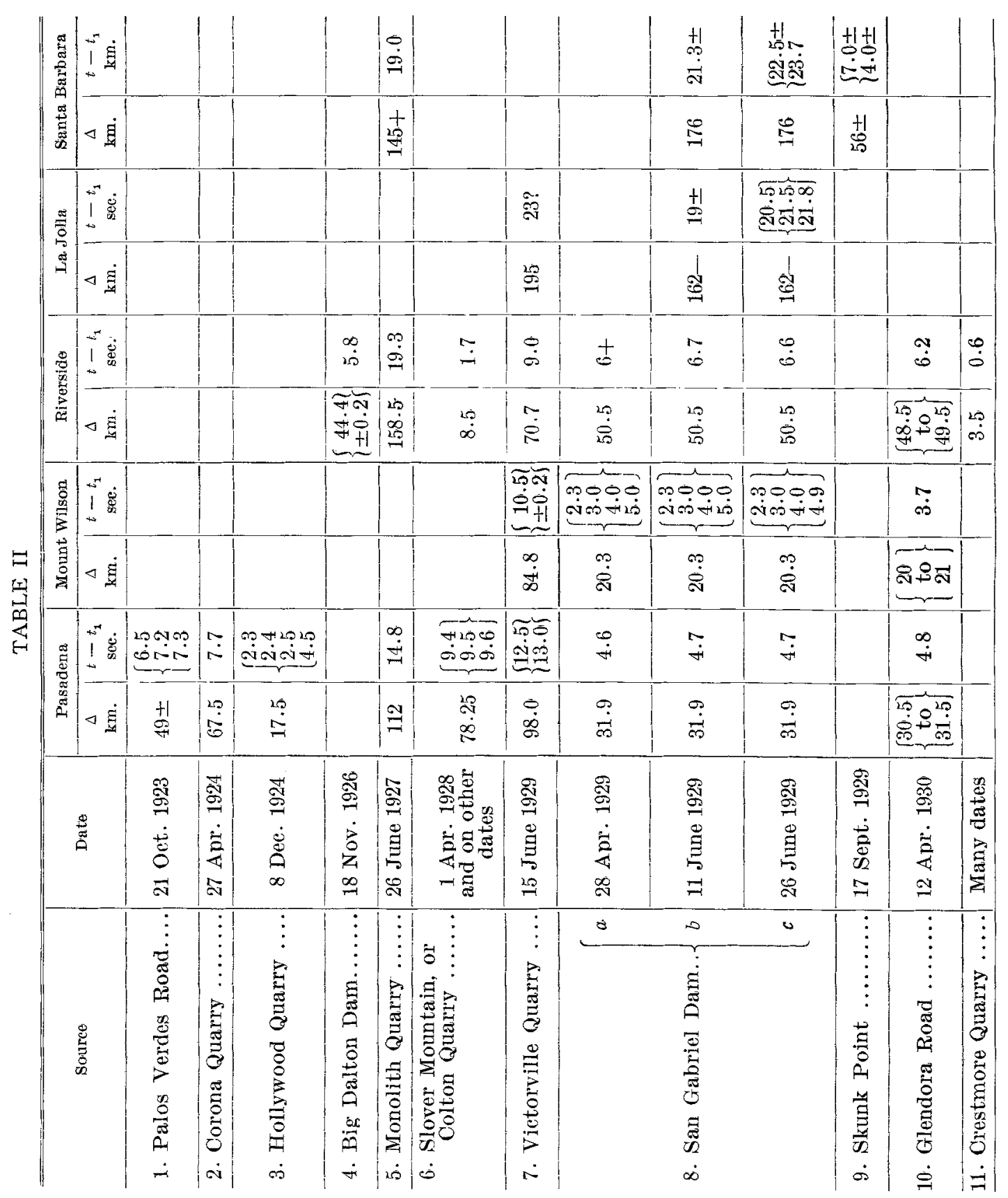




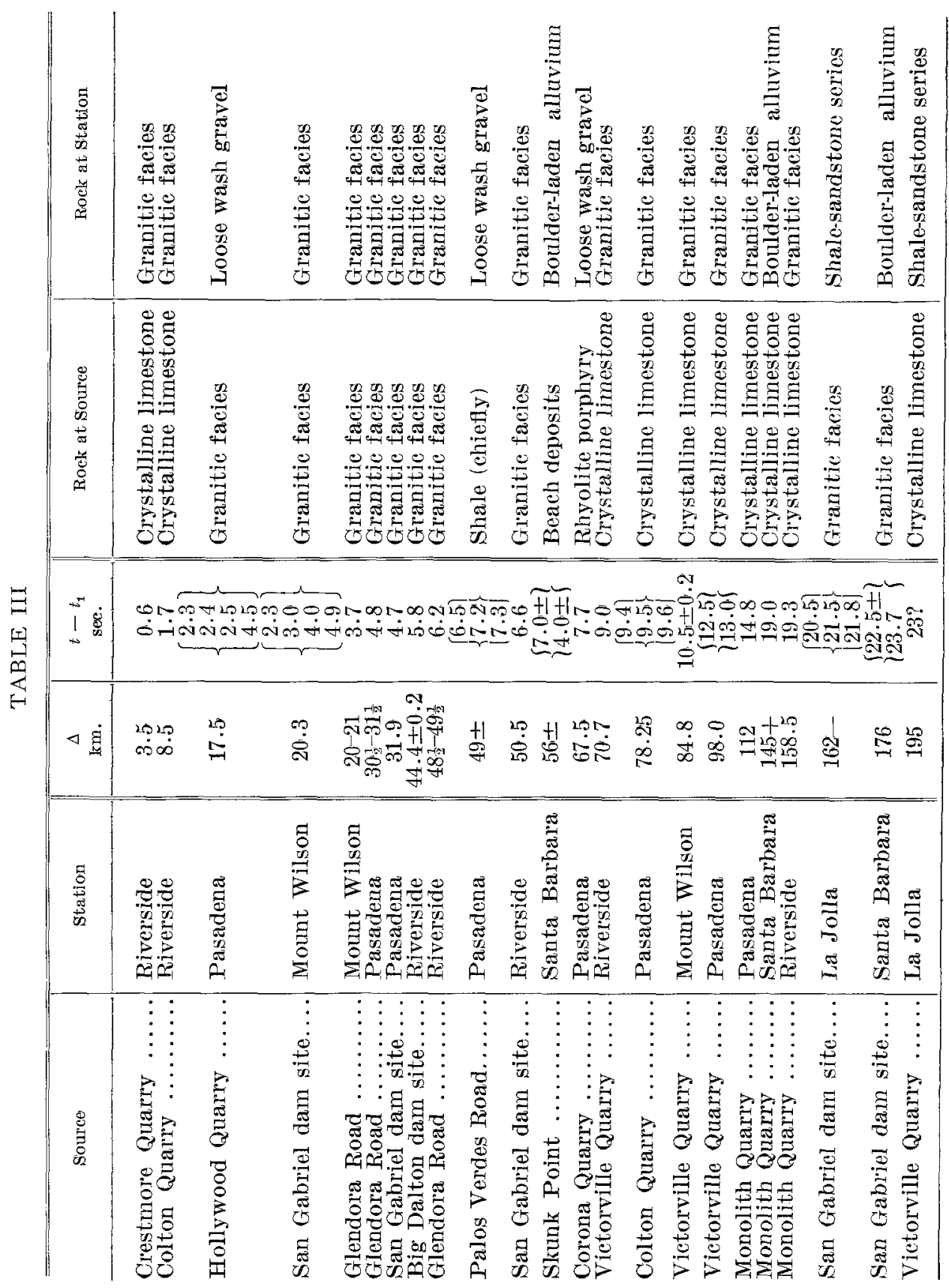


Subsequent blasts are summarized in part as follows:

3. Hollywood Quarry.

Date: December 8, 1924.

Location: Santa Monica Mountains east of Cahuenga Pass and north of Hollywood; "Hollywoodland" rock quarry.

Charge: stated to consist of 25,750 pounds of black powder.

Rock displaced: estimated at 45,000 to 50,000 tons.

Registered: at the temporary station, laboratories of Mount Wilson Observatory, Pasadena, at a distance of 19.25 kilometers (12 miles). Record shows considerable amplitude; legibility impaired by town and traffic disturbances.

The charge was fired in crystalline rock said to be granitic. A trough containing rocks of the younger geologic series extends to unknown depth between the source and the seismometers, which were installed above more than 245 meters ( $800+$ feet) of loose coarse wash gravel.

4. Big Dalton Dam.

Date: November 18, 1926.

Location: near Big Dalton flood control dam site.

Charge: stated to consist of 30 tons of high explosives.

Registered: clearly, with small amplitudes, at Riverside at a distance of $44 \mathrm{x} / 4$ kilometers $(27 \mathrm{I} / 2$ miles $)$.

The charge was fired in crystalline granitic rock and the seismometer piers rest directly on a granitic outcrop, but a valley fill more than 300 meters in depth intervenes, the upper part of which consists of loose, coarse, wash gravel.

5. Monolith Quarry.

Date: June 26, 1927.

Location: limestone quarry of the Monolith Portland Cement Company 3.5 kilometers (2.2 miles) northwest of Monolith in the Tehachapi Mountains.

Charge: stated to consist of 200,000 pounds of dynamite.

Rock displaced: estimated at 500,000 tons.

Registered: fairly well at Pasadena, Santa Barbara, and Riverside at distances of 112 kilometers ( 69.5 miles), $145+$ kilometers (90 miles ), and 158.5 kilometers (98.5 miles) respectively.

The wave-paths from the source to the stations at Pasadena and Riverside must have traversed crystalline rock for practically all, or all, of the distance. In traveling to Santa Barbara the waves at first traversed crystalline rock for nearly, or quite, half the distance, after which they passed through a series of folded Tertiary sedimentary rock of great 
vertical thickness (up to eight kilometers) proceeding at forty-five degrees, roughly, to the strike of the bedding and folding, and emerged finally through a small but unknown depth of heavy, boulder-laden, stream alluvium.

This was one of the cases when the date of firing was known in advance, but, on account of the large distances involved and lack of convenient facilities, no effort was made to determine precisely the instant of detonation.

6. Slover Mountain (or Colton) Quarry.

Date: April 1, 1928.

Location: limestone quarry of the California Portland Cement Company on the southwest side of Slover Mountain near Colton.

Charge: stated to consist of 85 tons of dynamite.

Rock displaced: estimated at 75,000 tons.

Registered: feebly at Pasadena at a distance of 78.25 kilometers (48.6

miles); and at Riverside distant 8.5 kilometers ( 5.25 miles) with amplitudes too large for use. However, many smaller blasts from this quarry have been registered clearly at Riverside.

The wave-path from the quarry to Pasadena passes beneath two large valley areas filled with wash gravels and alluvium to considerable depth, and also beneath a body of folded Tertiary sediments which extend to an unknown depth. However, crystalline rock outcrops at the surface at both the source and the station so it is possible, and probable, that the path traverses such a medium for the entire distance. The wave-path from the quarry to Riverside is wholly in crystalline rock, though a shallow trough of wash gravels and alluvium intervenes.

7. Victorville Quarry.

Date: June 15, 1929.

Location: limestone quarry of the Southwestern Portland Cement Company 10 kilometers ( 6.2 miles) north of Victorville.

Charge: 119,500 pounds of 30 per cent dynamite.

Rock displaced: estimated at 472,000 tons.

Registered: well and clearly at Riverside, Pasadena, and Mount Wlison, and with small amplitude at La Jolla, at distances of 70.7 kilometers (44 miles), 98.0 kilometers (61.0 miles), 84.8 kilometers (52.5 miles), and 195 kilometers (121 miles) respectively.

Except for relatively thin wash gravels at the surface, the wave-paths from the source to the stations traverse a complex of crystalline rocks for the most part, but in the case of La Jolla a minor amount of sedimentary rock may be encountered in troughs en route, and in the case 
of Mount Wilson the straight line passes in part through the air. In this connection it should be mentioned that the seismograms from Mount Wilson exhibit peculiarities.

The shock from this blast was taken to be a small local earthquake and an origin was located in the usual routine procedure, at a point about 6 kilometers ( 3.7 miles) north of its actual source on the surface. The closeness of this correspondence may be emphasized, particularly since an origin of some depth was presupposed when it was considered as a shock of natural origin. An engineer who visited the Seismological Laboratory gave information that the disturbance probably was due to this blast, and on further investigation this was found to be the fact.

8. San Gabriel dam site.

Date:

a) April 28, 1929

b) June 11,1929

c) June 26, 1929.

Location: site of the projected flood control dam at the forks of the San Gabriel River. Charge:

a) 46,150 pounds of black powder

b) 74,625 pounds of black powder

c) 122,950 pounds of black powder and 54,400 pounds of Herculite, equivalent to 20 per cent dynamite. (Also elsewhere given as 188,250 pounds of powder.)

Registered:

a) clearly at Pasadena and Mount Wilson, less well at Riverside

b) clearly at Pasadena, Mount Wilson, and Riverside, and less well at Santa Barbara and La Jolla

c) clearly at all stations in operation-Pasadena, Mount Wilson, Riverside, La Jolla, and Santa Barbara.

The distances from source to station are as follows:

Mount Wilson, 20.3 kilometers (12.6 miles) ; Pasadena, 31.9 kilometers (19.8. miles) ; Riverside, 50.5 kilometers ( 31.4 miles); La Jolla, 162- kilometers (100+ miles); and Santa Barbara, 176 kilometers (109.3 miles).

The wave-path in the case of Mount Wilson traverses granitic rock only, but the straight line from source to station passes in part through the air ; in the case of Pasadena it is confined to granitic rock, except for the improbable contingency that it may encounter the loose, wash gravel 
fill, known to be more than 245 meters $(800+$ feet) deep, which lies at the base of the Sierra Madre range. The same is true in the case of Riverside where the loose gravel and underlying alluvium reaches a depth of more than 300 meters (1,000 feet). In its course to La Jolla the wave-path at first traverses granitic rock, then it passes through, or beneath, a body of folded Tertiary sediments, later through, or beneath, the southwest flank of an anticlinal mass of considerable dimensions made up of Triassic, Cretaceous, and Tertiary rocks, and finally, through or beneath a region in which crystalline and sedimentary rocks alternate in an irregular way along the surface. However, it is not improbable that the wave-path in this case traverses granitic material for a large part, possibly all, of its way. En route to Santa Barbara, the wave-path at first traverses granitic rock for at least one-third of the distance; at some point at or more than one-third of the total distance from source to station, it must enter the great trough of folded Tertiary sediments and pass in a general way along the strike of both bedding and folding. These sediments extend to great vertical depth, estimated, or approximately measured, to be eight kilometers or more. In lithology and elastic properties these rocks, at the surface, vary in marked degree. Finally the wave must pass up through a small but unknown depth of heavy, boulder-laden alluvium to the instrument pier.

The date of firing of the blast on June 26th was known in advance, and an attempt was made to determine the instant of detonation very precisely, by direct telephone connection between the firing switch and the Seismological Laboratory. Unfortunately, through misunderstanding, the firing switch was closed before the giving of the pre-arranged signal, so this effort gave only a moderately good determination, which was checked independently by timing with rated watches made by Mr. E. C. Eaton, Chief Engineer of the Los Angeles County Flood Control organization, and assistants. When referred to the clock readings, these time observations yielded a time for the instant of firing good only to one or two seconds. We are glad to extend our thanks to $\mathrm{Mr}$. Eaton, and to the resident engineer at the dam site, for their assistance and the courtesies extended.

9. Skunk Point.

Date: September 17, 1929.

Location: Skunk Point, Santa Rosa Island.

Charge: 74-pound TNT mines, twelve in number, used by the United States Coast Guard in destroying a wrecked barge on the beach. 
Registered: at Santa Barbara only, at a distance of about 56 kilometers ( $35 \pm$ miles), as four groups of three disturbances each. The records exhibit peculiarities, and difficulty in interpretation is increased by local microseismic motion on this occasion.

The nature of the wave-path is in doubt. Ocean water, with a maximum depth exceeding 200 fathoms, obscures the surface for all but five kilometers $(3+$ miles $)$ of the distance from source to station. The beach sands at Skunk Point rest upon Quaternary sediments, and at the station a small but unknown depth of heavy, boulder-laden alluvium rests upon a great thickness of folded Tertiary sediments.

10. Glendora Road.

Date: April 12, 1930.

Location: a point on the so-called Glendora Road east of the San Gabriel Canyon, in N.E. quarter of Section 5, T. 1 N., R. 9 W., San Bernardino Base and Meridian.

Charge: stated to consist of three tons of black powder fired in road construction work.

Registered: well at Mount Wilson, Pasadena, and Riverside at distances of 20 to 21 kilometers ( 12.5 to 13 miles), 30.5 to 31.5 kilometers ( 19 to 19.5 miles), and 48.5 to 49.5 kilometers ( 30 to 30.5 miles), respectively.

Information at hand states that a blast of this magnitude was fired at the location mentioned, on some date "in March or April" of 1930. There is little doubt that this disturbance is the blast in question, for such a shock should be registered and nothing else has been found to correspond. Also this was judged to be the record of a blast at the time of its registration, but no information about it was obtained until much later.

On this occasion the wave-paths were confined to granitic rock, except as modified by deep, loose, wash gravel en route to Pasadena and Riverside.

11. On numerous dates extending from late in 1926 down to the present, small blasts at the Crestmore limestone quarry of the Riverside Cement Company, distant about 3.5 kilometers (2.2 miles) from the Riverside station, have been registered clearly there. In such instances the wave-paths have traversed crystalline rock throughout, unless the alluvium of the Santa Ana River extends deep enough to interfere.

\section{Discussion}

In general the seismograms of these blast shocks closely resemble records of small earthquakes of near-by origin, though peculiarities are 
noticeable in some cases. The records exhibit an interval of preliminary motion of smaller amplitude, in which complications are often seen, followed by motion of larger amplitude which usually begins abruptly and thus suggests the arrival of an $S$-phase. Therefore, for preliminary purposes, the beginning of registered motion was interpreted as $\bar{P}$, and the abrupt beginning of motion of larger amplitude as $\bar{S}$. Measurements were made on this basis, which in some instances had to be revised as a result of study and discussion of the results. The time-intervals from the beginning of motion to the beginning of certain other waves were measured also. Most of these measurements appear in Tables II and III as $t-t_{1}$, where $t_{1}$ is the time of beginning of discernible motion. Hence $t-t_{1}$ equals $\bar{S}-\bar{P}$, as interpreted, in many instances but not in all. A few points not included in the table are shown on the diagram, Figure 2.

\section{VELOCITIES}

However, because of the peculiarities and complications exhibited by the seismograms, because the wave-speeds in this region had not been determined from previous observations, and because the structure of the upper portion of the earth crust in this region is not yet known with definiteness or certainty, it is clear that the identification. of phases needs to be confirmed or verified by correlation with measured transmission times. Data suitable for this are available in three cases: (1) the time of firing of the Corona blast is known with high accuracy; (2) in the case of the Victorville blast the time marks on the seismograms written at Pasadena and at Riverside can be compared very accurately by reference to radio code signals also registered parallel with the time-marks at both stations; and (3) in the case of the major blast at the San Gabriel dam site on June 26, 1929, the time-marks at Pasadena and Riverside can again be compared in the same way.

In order to derive wave-speeds in cases 2 and 3 it is necessary to assume that the beginnings of certain waves, or wave-groups, at Pasadena and at Riverside represent the arrivals of corresponding phases. No such assumption is required in case 1.

In the case of the Corona blast the most conspicuous impulse, which normally would be identified as $\bar{S}$, arrived at Pasadena 21.0 seconds after the time of firing. This measurement is considered to be accurate within 0.1 second. The distance from source to station is 67.5 kilometers (42 miles) with an error probably not exceeding 0.1 kilometer ( 0.06 mile). Consequently the calculated velocity of 


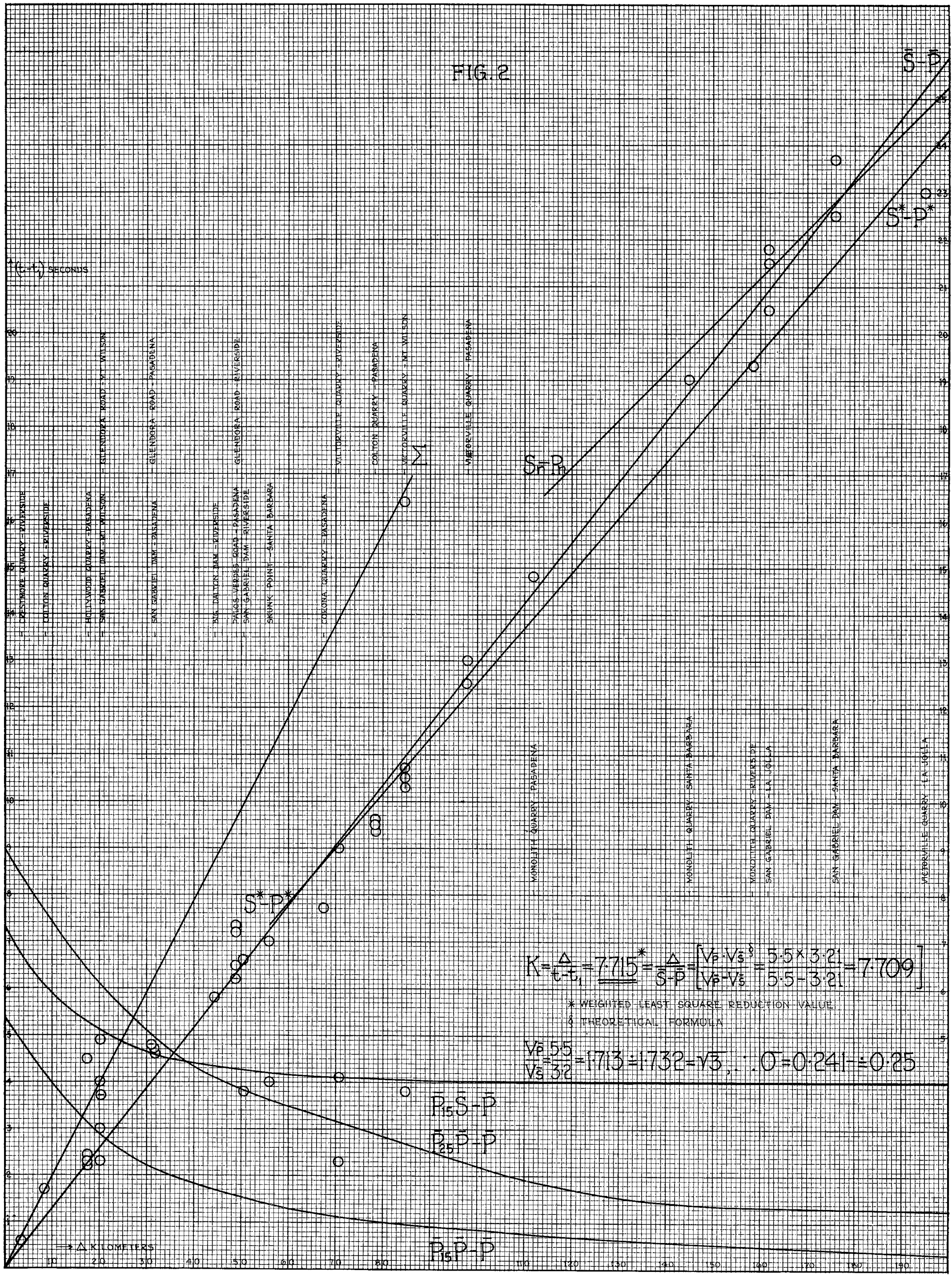


the wave represented by this impulse is 3.21 kilometers per second with an error not exceeding 0.02 kilometer per second. Therefore, with high probability, this phase may be taken as $\bar{S}$.

On the date of the Corona blast the line of the seismogram was blurred slightly and almost continuously by town and traffic disturbance (at the temporary experimental station at the laboratories of the Mount Wilson Observatory). The apparent velocity of the wave-motion which first emerges from this disturbed line is 5.08 kilometers per second. Consequently this cannot well be the phase, $\bar{P}$, corresponding directly to the phase, $\bar{S}$, which is considered to be well identified. This is discussed further on page 42 .

In the case of the Victorville blast the first motion discernible at Riverside arrived there 5.2 seconds earlier than the arrival at Pasadena of the first motion discernible there. This measurement may be in error by as much as 0.1 second. By measurement Riverside is 27.3 kilometers (17 miles) nearer the source than Pasadena is, but the error in this measurement, which is not easily estimated, may perhaps reach one kilometer ( 0.6 mile) though it probably is less. On this basis the calculated wave-speed is 5.25 kilometers per second, with an error not exceeding 0.3 kilometer per second. This mean velocity appears somewhat low for $\bar{P}$, as will be seen farther on.

In the case of the transversal $\bar{S}$ wave in the Victorville blast records, only one wave-beginning is recognized on the seismograms written at Riverside which may be identified with this phase. At Pasadena, however, the seismograms permit two alternative readings for this phase, separated by 0.5 second, and either of the resulting intervals, 8.7 seconds and 9.2 seconds, may be in error by 0.1 second. The corresponding calculated wave-speeds are, in the first case 3.15 kilometers per second with an error not exceeding 0.15 kilometer per second, and in the second case 2.97 kilometers per second with an error not exceeding 0.14 kilometer per second. From this it appears that the phase identified at Riverside, and the earlier indication at Pasadena, represent the $\bar{S}$ phase. In such case the mean velocity appears somewhat low, as will be seen farther on.

In the case of the major San Gabriel dam blast the first impulse recorded at Pasadena arrived there 3.3 seconds before the first motion registered at Riverside reached there. This measurement may be in error by as much as 0.1 second. Pasadena is 18.6 kilometers ( 11.5 miles) nearer the source than Riverside. This measurement is in error by considerably less than 0.1 kilometer $(0.06$ mile $)$. Consequently, the calcu- 
lated value of the velocity is 5.67 kilometers per second with an error not exceeding 0.2 kilometer per second. From this it appears that the waves which first arrived are $\bar{P}$. This value may be compared with the $5.25 \pm 0.3$ kilometers per second ${ }^{1}$ which was derived in the case of the Victorville blast. Assuming that the velocities are fairly constant throughout the region, and that the phase $\bar{P}$ arrived at both Pasadena and Riverside from both the Victorville and the San Gabriel dam blasts, the velocity of $\bar{P}$ must lie between 5.47 and 5.55 kilometers per second. Consequently this velocity is $\mathbf{5 . 5}$ kilometers per second to the nearest tenth.

The velocity of $\bar{S}$, in the case of the San Gabriel Dam blast, cannot be derived-for the following reason. The phase at Pasadena which naturally would be identified with $\bar{S}$ arrived there 5.2 seconds earlier than the similar phase arrived at Riverside. This yields a calculated velocity of 3.6 kilometers per second which manifestly is too high when compared with all the other data available. Also, with this identification, the $\bar{S}-\bar{P}$ interval measured from the Pasadena records is manifestly too long for the known distance. In other words, the apparent $\bar{S}$ at Pasadena arrived too late, making the apparent $\bar{S}-\bar{P}$ too great, while the apparent $\bar{S}_{\text {Riverside }}-\bar{S}_{\text {Pasadena }}$ is too short, leading to a too high value for the velocity of $\bar{S}$. This phase at Pasadena which resembles $\bar{S}$ appears to be a reflected wave which is discussed farther on, but the phase taken to be $\bar{S}$ at Riverside probably is identified correctly.

From the foregoing discussion it is clear that the best velocity for $\bar{S}$ is that derived from the Corona blast, namely, 3.21 kilometers per second with an error not exceeding 0.02 kilometer per second as calculated. This value, therefore, is adopted for further discussion.

No similarly accurate value for $\bar{P}$ has yet been obtained.

Nevertheless the data afford one further method for determining a velocity for $\bar{P}$, namely, from consideration of the reduced $\bar{S}-\bar{P}$ observations together with the adopted velocity for $\bar{S}$.

Measured values of $t-t_{1}$ were plotted as ordinates for the twentythree available independent distances, $\Delta$, taken as abscissas. Mere inspection disclosed that most of the points fell along a straight line through the origin with surprisingly small departures, even though the data are uneven in quality. Selecting those points which seemed most reasonable, taking all the facts into consideration, and using one $t-t_{1}$ interval for each $\Delta$, an unweighted least-squares solution was made to determine the

I The \pm symbol with the number following it denotes "limit of error" and not "probable error." The same usage is followed throughout the paper. 
line represented by $\frac{\Delta}{t-t_{1}}=k$. On this basis $k$ was found to be 7.89 . It was recognized that some measurements of $t-t_{1}$ were of better quality than others, so a weighted least-squares solution was made, for all the values of $\Delta$, which yielded $k=7.825$.

However, as the study developed it became apparent that measured $t-t_{1}$ intervals for distances less than 40 kilometers (25 miles) exhibited peculiarities; and, from the beginning, it was clear that at distances greater than 150 kilometers (93 miles) the measured $t-t_{1}$ intervals were somewhat wanting in accuracy, and that complications due to the arrival of other phases may introduce confusion in this distance range. Consequently, a weighted least-squares reduction, based on carefully revised measurements of $t-t_{1}$ and of $\Delta$ between 40 and 150 kilometers ( 25 and 93 miles) was carried through. From this a value of $k=7.715$ was obtained. This value, therefore, is adopted for calculation, with the understanding that no claim is made for high accuracy in the case of the last digit.

Theoretically, assuming rectilinear propagation of the seismic waves, $k=\frac{V_{\bar{S}} V_{\bar{P}}}{V_{\bar{P}}-V_{\bar{S}}}$. Hence, $V_{\bar{P}}=\frac{k V_{\bar{S}}}{k-V_{\bar{S}}}=5.50$ kilometers per second with an error probably not exceeding 0.06 kilometers per second.

This gives for $V_{\bar{P}}$ slightly wider limits of error than those found above from differences in arrival times at Pasadena and Riverside, but the mean value is substantially the same. It is concluded, therefore, that $V_{\bar{p}}=5.5$ kilometers per second to the nearest tenth.

\section{Other Phases}

It will be recalled that the first motion discernible on the Pasadena records of the Corona blast arrived later than the expected time for $\bar{P}$, its transmission time being 13.3 seconds. This record was studied some time ago by Byerly ${ }^{2}$ and compared by him with the record of a natural earthquake registered at Tacubaya from about the same distance. Byerly suggested that "The first arrival on the Pasadena record .... (has) the travel-time of Jeffreys' $P$ which has been twice refracted at the base of the granitic layer (or perhaps reflected at this surface, since at this distance the travel-times for these two distances are nearly the same), if the granitic layer has a thickness of fifteen kilometers and Jeffreys'

${ }^{2}$ Bulletin of the National Research Council, No. 61, July, 1927, pp. 88-90. 
velocities in the two upper layers hold." We confirm Byerly's finding; and, further, taking our velocity of 5.5 kilometers per second for the upper layer (which may be regarded as a minimum) and assuming a velocity of 6.2 or 6.3 kilometers per second for the second layer, we compute depths of 14.4 kilometers (8.95 miles) and 14.7 kilometers ( 9.1 miles) for the surface of refraction, while with our velocity of 5.5 kilometers per second a surface of reflection is computed at a depth of 14.1 kilometers ( 8.75 miles). On the other hand, taking 5.6 kilometers per second as the velocity in the upper layer, following Jeffreys' earlier determination, these values become 15.7 kilometers and 15.8 kilometers in the case of refraction and 15.7 in the case of reflection. With Jeffreys' later velocity of 5.4 kilometers per second these computed depths obviously will be smaller than with our value. Therefore, in any case, taking this wave-arrival to be either $P^{*}$, or a reflected $\bar{P}$ phase, the thickness of this upper layer is indicated by this observation to be about 15 kilometers (9.3 miles) with a small error of uncertain amount.

Assuming, as a working hypothesis, a thickness for the upper layer of 15 kilometers ( 9.3 miles) and for the second layer of 25 kilometers (15.5 miles) and taking Jeffreys' velocities for all the layers, curves were computed and checked for $S^{*}-P^{*}$ (and for $S_{n}-P_{n}$ ). These curves would be changed only slightly if our velocities for the upper layer were to be substituted. When these were laid down on the plot for $t-t_{1}$ against $\Delta$, it was seen that none of the aberrant points $\left(t-t_{1}\right)$ corresponded any better with the $S_{n}-P_{n}$ curve than with the $\bar{S}-\bar{P}$ curve. At the greater distances, however, at least two of the points $\left(t--t_{1}\right)$ corresponded much better with the $S^{*}-P^{*}$ curve than with the $\bar{S}-\bar{P}$ curve. No emphasis can be placed upon this finding, but it suggests that at distances greater than, say, 150 kilometers (93 miles) the $P^{*}$ phase in some instances preceded the $\bar{P}$ phase in arrival at the stations. The failure to find the $P_{n}$ phase, corresponding to a wave which had suffered refraction four times between source and stations, may be due to the small original energy of these artificial disturbances and the relatively great losses in transmission. On the other hand, within the distance range 150-200 kilometers, as the diagram, Figure 2, shows, it is more difficult to distinguish $P_{n}$ from $\bar{P}$ than to distinguish $P *$ from $\bar{P}$.

There remain a number of $t-t_{1}$ measurements which do not correspond with any of the phases, or phase-intervals, so far discussed. In the case of the San Gabriel Dam blast as registered at Pasadena, it has been mentioned that the phase which resembles $\vec{S}$ arrived too late to be identified satisfactorily as such. It was suggested that this may have been 
due to reflection. This phase follows $\bar{P}$ by 4.7 seconds where the distance is 31.9 kilometers ( 19.7 miles). In the case of the Glendora Road blast registered also at Pasadena at a distance of about 31 kilometers (19.5 \pm miles), there appeared to be a similar phase which followed $\bar{P}$ by 4.8 seconds. Assuming, as a working hypothesis, that these late arrivals correspond to reflected waves, certain trial computations were made.

It was soon seen that no simple reflections could be made to correspond, taking velocities in the ratio of $1.73\left(V_{\bar{p}}=5.55, V_{\bar{s}}=3.21\right)$, and a depth of 15 kilometers $(9.3$ miles) for the reflecting surface. It was found, however, that the assumption of a longitudinal wave reflected at a depth of fifteen kilometers, and transformed by reflection into a transversal wave, with the given velocities and velocity-ratio, fits the observations fairly well. Further, a curve $\vec{P}_{1 \bar{s}} \bar{S}-\bar{P}$, drawn on this basis on the diagram, Figure 2, is seen to pass not only close to these points, but also near to several others within the range of distance between 10 and 90 kilometers ( 6.2 and 56 miles). Theoretically, however, the energy arriving at the stations by this route should be small, so the apparent correspondence is presented for consideration without emphasis. It was found further that the curve for a longitudinal wave reflected as such at a depth of twenty-five kilometers, shown on the diagram as $\bar{P}_{25} \bar{P}-\bar{P}$, Figure 2 , also passed fairly close to the points $\left(t-t_{1}\right) 4.7$ and 4.8 at a distance of 31 to 32 kilometers; but this curve elsewhere passes close to only one other point. A single longitudinal reflection from a greater depth, as thirty kilometers, fits less well. A curve $\bar{S}_{15} \bar{S}-\bar{S}$, also plotted but not published, failed to correspond with any of these points. Assumption of a different depth for the reflecting surface will make no improvement in this case.

A wave traveling with a velocity slower than that of $\bar{S}$ is indicated by phases on the records at Riverside of blasting at Crestmore and Colton, as may be seen from the diagram, Figure 2. On this diagram a straight line through the origin, marked $\Sigma$, which passes very close to these phasepoints, also approaches very closely the $t-t_{1}$ measurement of a conspicuous impulse on the Mount Wilson seismograms of the major San Gabriel Dam blast. If it is assumed that these points represent an actual phase, a velocity of rectilinear propagation can be computed which proves to be about 2.6 kilometers per second. Various hypotheses have been considered to account for this phase, but none have afforded a satisfactory explanation. It will be noted that the $\Sigma$ line on the diagram passes close to a point representing a late arrival at Mount Wilson in the case of the 
Victorville blast. The absence of any intermediate points, however, suggests that this is simply a coincidence.

\section{SUMMARY}

As an outcome it is found:

1. For surface paths, or paths of slight depth in the upper ("granitic"Jeffreys) layer in this region the velocities of the longitudinal and transversal waves are, respectively, 5.5 kilometers per second and 3.21 kilometers per second, very nearly. (With natural shocks, originating below the surface, slightly higher values probably may be expected) ;

2. A thickness of the upper layer of about 15 kilometers, or of about 25 kilometers, is indicated;

3. No waves have been identified as $P_{n}$ or $S_{n}$ with any definiteness;

4. Waves identified as $P^{*}$ and $S^{*}$ appear to be registered in some cases. (The data are consistent with a velocity of 6.2 to 6.3 for longitudinal waves in the second layer);

5. In several cases readings of apparent phase-intervals suggest the existence of a longitudinal wave reflected as a transversal wave at a depth of about 15 kilometers;

6. In one or two cases readings of apparent phase-intervals suggest the possibility of a longitudinal wave reflected as such at a depth of 25 kilometers ;

7. At the smaller distances there is some indication of a wave traveling with a velocity of 2.6 kilometers per second.

The need of further data to extend and test these conclusions is apparent.

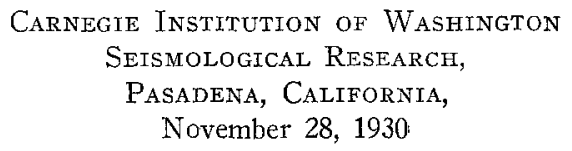

